\title{
Measuring the gluon distribution in nuclei at an Electron-Ion Collider
}

\author{
Matthew A. C. Lamont \\ Brookhaven National Lab, Upton, NY, 11973, USA \\ DOI: http://dx.doi.org/10.3204/DESY-PROC-2014-04/44
}

\begin{abstract}
Despite the successes of the HERA accelerator, where much information was gained on the structure of the nucleon, data on the structure of the nucleus at moderate-to-small $x$ remains elusive, as only fixed-target high- $x$ data currently exist. The small- $x$ region, however, is of great interest. The nucleon structure in this region is dominated by gluons which show a rapid rise with decreasing $x$. At low- $x$, this growth must be tamed and the gluon distribution will be saturated. However, only tantalising hints of this have been observed so far. Therefore, the construction of an Electron-Ion Collider (EIC), colliding polarised electrons with polarised protons and also a wide variety of nuclei, will allow an exploration of the region of small- $x$ in great detail, answering questions on both the spatial and momentum distributions of gluons and sea quarks in nuclei. In particular, the saturation region is more accessible in nuclei due to the amplification of the saturation scale with nuclear size $\left(Q_{S} \propto \mathrm{A}^{1 / 3}\right)$. In this paper I present the current status of measuring the gluon distribution in nuclei in $e+\mathrm{A}$ collisions at an EIC.
\end{abstract}

\section{Introduction}

As the HERA measurements of structure functions showed, at small $x$, the gluon and seaquark distributions dominate the structure of the nucleon [1]. In fact, if the structure functions continued to grow untamed with decreasing $x$, through a process of hard gluons splitting into softer gluons, then the cross-section would become larger than the black-disk limit. Therefore, this growth must be tamed at small $x$, when soft gluons can recombine into harder gluons. Saturation is achieved when these two processes match each other. This saturation phenomena is expected to be universal, appearing in both nucleons and nuclei. However, its effect is amplified in nuclei, where the saturation scale is expected to grow with nuclear size $\left(Q_{S} \propto\right.$ $\left.\mathrm{A}^{1 / 3}\right)$. Therefore, saturation effects should be visible at higher values of $x$ than in nucleons. A review of saturation physics can be found in the literature [2]. A knowledge of this regime is of vital importance to understanding the underlying physics which governs the initial conditions of heavy-ion collisions at both RHIC and in particular, the LHC, where particle production is dominated by gluons from this unknown region. To that end, eRHIC is a machine that is being designed at Brookhaven National Lab that utilizes the current \$2B hadron facility of RHIC and would provide $e+\mathrm{A}$ collisions at high energy and high luminosity [3]. eRHIC would also provide polarized electrons and protons for the study of the spin structure of nucleons.

In this paper, I will not discuss the spin capabilities of an electron-ion collider, but rather focus on measurements that can be made in $e+\mathrm{A}$ collisions which will provide further insight 


\section{MEASURING THE GLUON DISTRIBUTION IN NUCLEI AT AN ELECTRON-ION COLLIDER}

into the structure of nuclei. For a detailed review of both the spin capabilities and a more in-depth coverage of the $e+\mathrm{A}$ physics than is allowed in this manuscript, please refer to the EIC White Paper [4].

\section{Structure Functions}

One of the first measurements that will be made in nuclear DIS collisions is of the cross-section as a function of $\left(\mathrm{x}, Q^{2}\right)$. Although this is one of the easiest measurements to make, it contains a lot of important information. This cross-section can be written in terms of structure functions:

$$
\sigma_{r}\left(x, Q^{2}\right)=F_{2}^{A}\left(x, Q^{2}\right)-\frac{y^{2}}{Y^{+}} F_{L}^{A}\left(x, Q^{2}\right)
$$

The two structure functions of importance are $F_{2}$ and $F_{L}$, which reflect the quark and gluon momentum distributions respectively. In order to measure $F_{L}$ directly, it is necessary to have high-statistics datasets over a number of energies. This was not possible at HERA and hence the gluon distribution in nucleons was inferred from the scaling violation of the $F_{2}$ distribution [1].
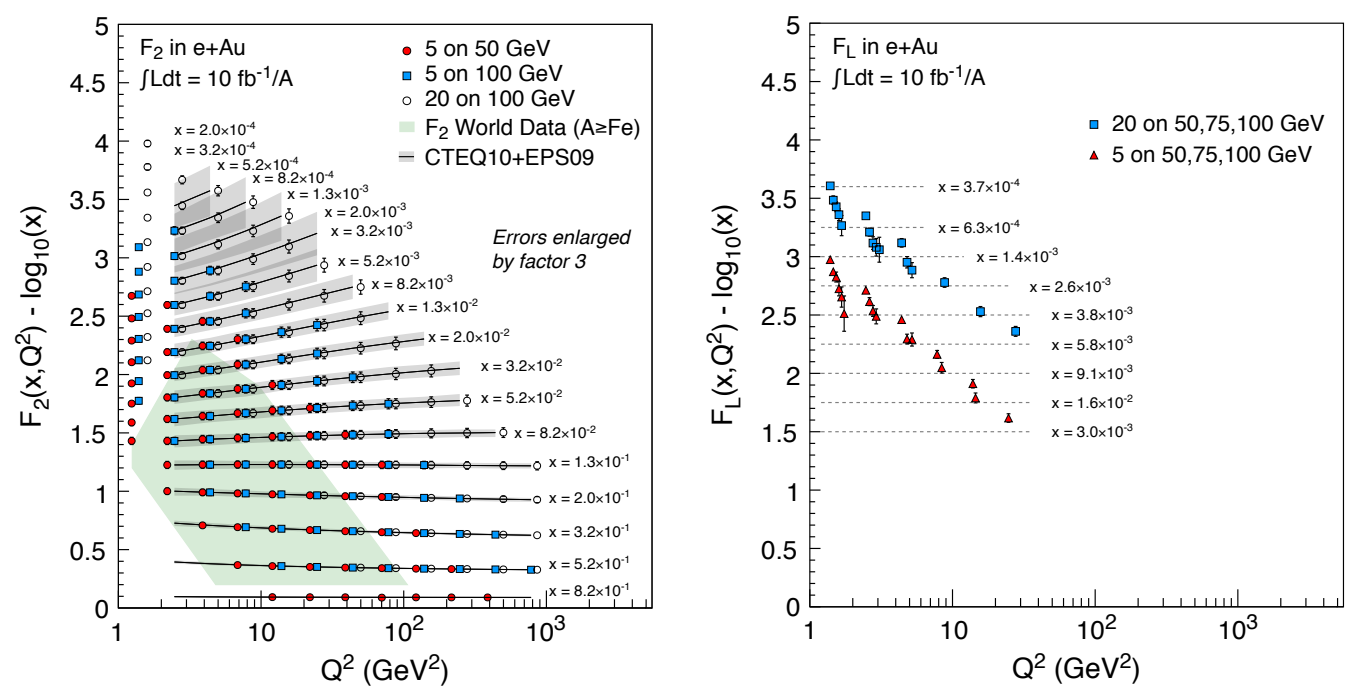

Figure 1: The $F_{2}$ (left) and $F_{L}$ (right) distributions at eRHIC for $e+\mathrm{Au}$ collisions. The uncertainties on the pseudo-data are overwhelmingly systematic and represent an integrated luminosity of $10 \mathrm{fb}^{-1} / \mathrm{A}$.

The left side of Figure 1 shows the $\left(x, Q^{2}\right)$ coverage of the $F_{2}$ distribution according to the pseudo-data generated for three different eRHIC energies. These pseudo-data were generated using PYTHIA with EPS09 NLO PDFs and represent $10 \mathrm{fb}^{-1} / \mathrm{A}$ of data, corresponding to approximately 6 months running time. The highest energy (20x100 GeV beams) extend the phase-space to the lowest $\left(x, Q^{2}\right)$ region while the lowest energy ( $5 \times 50 \mathrm{GeV}$ beams) extends this measurement to high- $x$. Also shown on the plot are the EPS09 predictions and their associated uncertainties, together with existing data from fixed target $e+\mathrm{A}$ collisions. At small- $x$, these 
uncertainties are very large and the data from eRHIC will have a significant impact on the EPS09 uncertainties. For clarity, both the errors coming from EPS09 and the pseudo-data have been enlarged by a factor of 3 .

The right side of Figure 1 shows the corresponding $F_{L}$ measurement. The $F_{L}$ data were extracted using a Rosenbluth Separation analysis technique where, for each $\left(x, Q^{2}\right)$ bin, a minimum of 3 data points were fit and were required to have a separation of at least 0.1 units of $y^{+}$. This technique, however, leads to large uncertainties and these are represented in the plot. Although these are significant, this will be a first measurement of $F_{L}$ in nuclei.

\section{$3 \quad$ Diffraction in $e+A$ collisions}

One of the interesting results to come out of HERA was that a large fraction of events (10-15\%) were diffractive. That is, in a significant fraction of collisions at very high energies, the nucleon stayed intact. Whilst this, in and of itself, is a large fraction of events, it is predicted that in nuclei, where saturation effects may be observed at eRHIC, this number could be a factor of 2 or 3 higher.

One of the most promising methods for observing saturation in diffractive collisions, other than the cross-section, is that of vector meson production. Figure 2 shows the cross-section for vector meson production in both coherent and incoherent diffractive collisions, as a function of the Mandelstam variable $t$, where the uncertainties correspond to an integrated luminosity of $10 \mathrm{fb}^{-1} / \mathrm{A}$. Note that the $Q^{2}$ range is above the photo-production threshold. This is shown for both the $\mathrm{J} / \psi$ and the $\phi$ vector mesons. Both of these distributions were generated using the SARTRE MC event generator which has recently been developed for diffractive physics in $e+\mathrm{A}$ collisions [5].
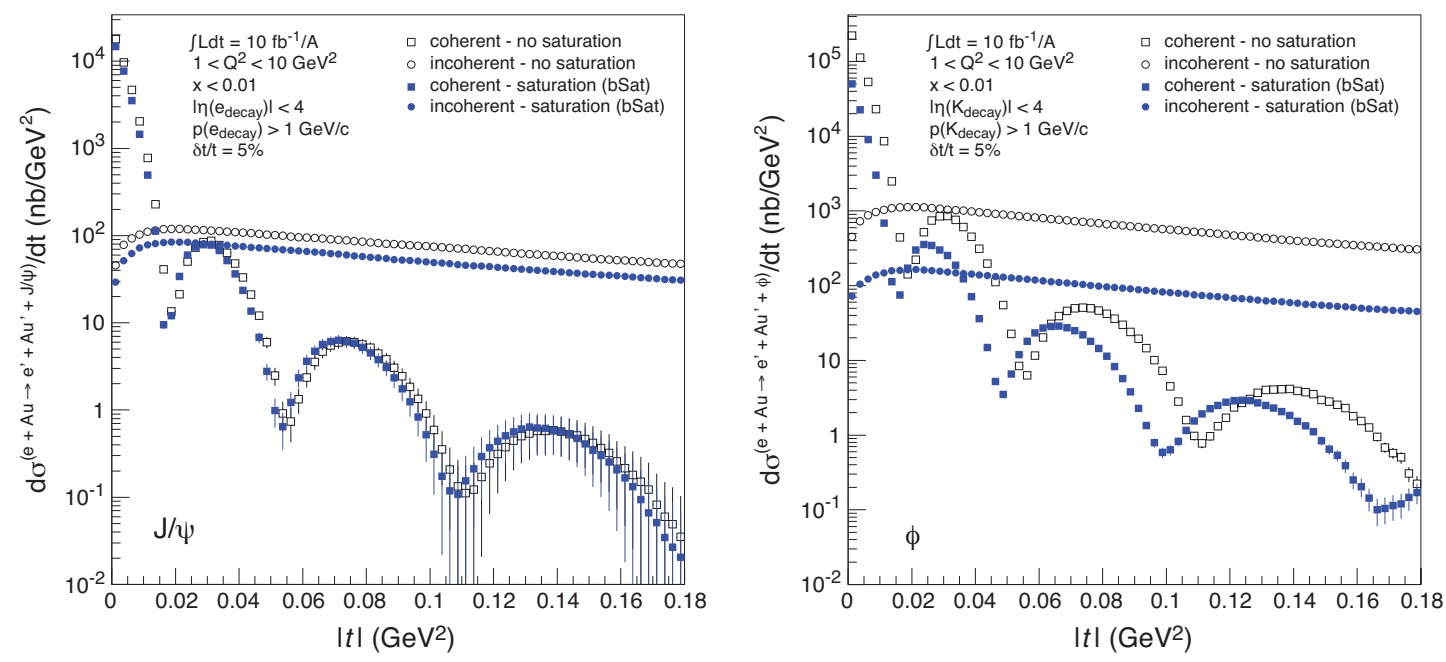

Figure 2: The coherent and incoherent diffractive distributions for the $\mathrm{J} / \psi$ (left) and the $\phi$ (right) vector mesons for an integrated luminosity of $10 \mathrm{fb}^{-1} / \mathrm{A}$, respectively. All distributions were calculated using the SARTRE event generator.

This shows that in the case of the $\mathrm{J} / \psi$, then there is very little difference between the 


\section{MEASURING THE GLUON DISTRIBUTION IN NUCLEI AT AN ELECTRON-ION COLLIDER}

distributions for the saturated and unsaturated case. However, for the $\phi$, there is a very significant difference. This comes about because of the size of the wave-functions, where the $\phi$ wave function is much larger than that of the $\mathrm{J} / \psi$ and hence it is more sensitive to saturation effects. In fact, one can take these distributions and perform a Fourier Transform to obtain the input source distribution. Figure 3 shows examples of this for the saturated and unsaturated case for both the $\mathrm{J} / \psi$ and the $\phi$ mesons, which plots both the input Woods-Saxon distribution and the Fourier Transform of the coherent diffractive distribution. As can be seen in the figure, there is little difference between the cases for the $\mathrm{J} / \psi$ whereas there is a large difference in the two scenarios for the $\phi$ meson.
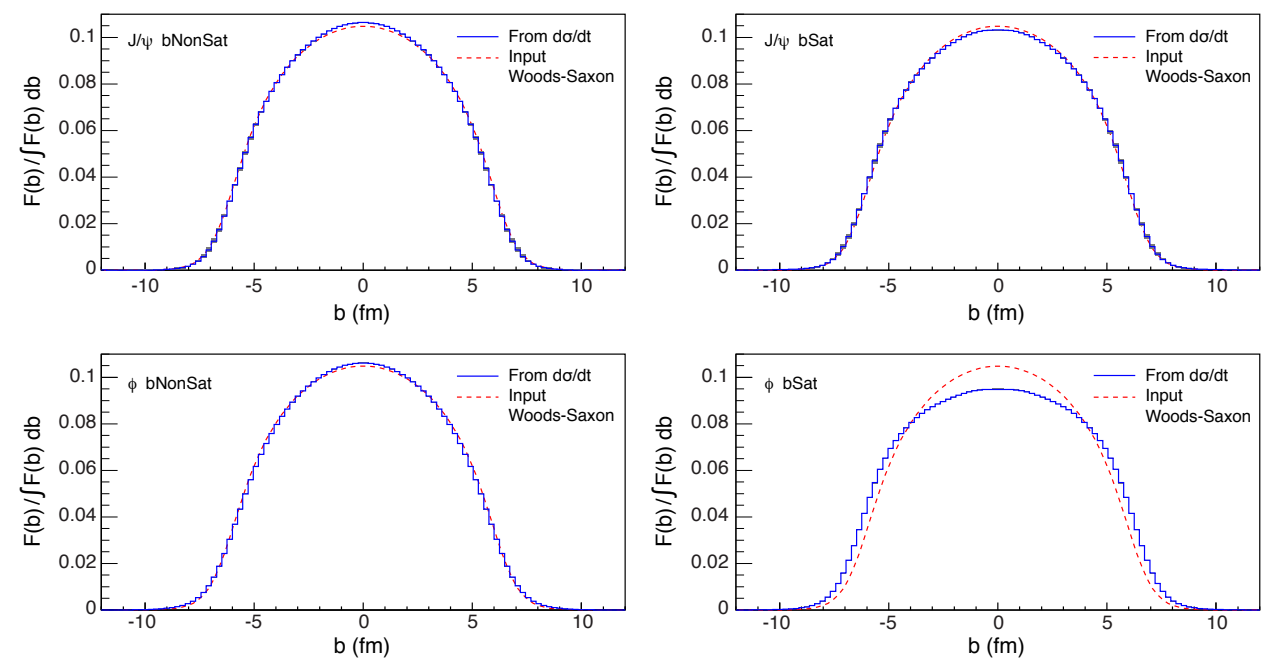

Figure 3: The Fourier Transform of the coherent diffraction distribution in the case of a saturated and unsaturated wave function for the $\mathrm{J} / \psi$ (top) and the $\phi$ (bottom) vector mesons presented in Figure 2. Also shown in each case are the input Woods-Saxon distributions.

Without an Electron-Ion Collider, these measurements cannot be made. Therefore, it is imperative that if we are to more fully understand the par tonic structure of the nucleus, an electron-ion collider is built.

\section{References}

[1] S. Aid et al., (H1 Collaboration) Phys. Lett. B354 (1995) 494-505.

[2] J. Jalilian-Marian and Y. V. Kovchegov, Prog. Part. Nucl. Phys. 56, 104 (2006).

[3] E.-C. Aschenauer et al., ArXiv:1409.1633.

[4] A. Accardi et al., ArXiv:1212.1701.

[5] T. Toll and T. Ullrich, Phys. Rev. C 87, 024913 (2013). 